\title{
Socioeconomic differentials in recurrent ischaemia and mortality after acute myocardial infarction
}

\author{
K Barakat, S Stevenson, P Wilkinson, A Suliman, K Ranjadayalan, A D Timmis
}

\begin{abstract}
Objective-To examine the influence of socioeconomic deprivation on case fatality following acute myocardial infarction.

Design-Prospective cohort observational study.

Setting-General hospital.

Patients-1417 white and south Asian patients admitted with acute myocardial infarction between January 1988 and December 1996, and classified by the Carstairs socioeconomic deprivation score of the enumeration district of residence.

Main outcome measures-30 day and one year survival.

Results-There was little variation across deprivation groups in age, sex, or smoking status, though a higher proportion of patients from more deprived enumeration districts were diabetic and of south Asian origin, and a higher proportion of them developed $\mathrm{Q}$ wave infarction and left ventricular failure. There was no appreciable variation in clinical treatment with deprivation. Patients from more deprived enumeration districts had a higher risk of recurrent ischaemic events (death, recurrent myocardial infarction, or unstable angina) over the first 30 days: event free survival (95\% confidence interval (CI)) of the most deprived quartile was 0.79 (95\% CI 0.74 to 0.83 ) compared with 0.85 (95\% CI 0.80 to 0.88 ) in the least deprived quartile. The unadjusted hazard ratio corresponding to an increase from the 5th to 95 th centile of the deprivation distribution was 1.54 (95\% CI 1.02 to 2.32), and 1.59 (95\% CI 1.03 to 2.44) after adjustment for age, sex, racial group, diabetes, acute treatment with thrombolysis and aspirin, and left ventricular failure. Survival from 30 days to one year, however, did not show a socioeconomic gradient (hazard ratio adjusted for the same variables was 1.07 (95\% CI 0.68 to 1.70$)$ ).

Conclusions-In patients hospitalised with acute myocardial infarction, there is a strong association between early recurrent ischaemic events and socioeconomic deprivation that is not accounted for by clinical presentation or treatment. This association appears to be attenuated over time.

(Heart 2001;85:390-394)
\end{abstract}

Keywords: acute myocardial infarction; socieconomic deprivation; survival

Inequalities in health driven by socioeconomic deprivation continue to undermine the wellbeing of large sections of the population. ${ }^{12}$ This is particularly true of coronary heart disease, which remains the major cause of premature death in most industrialised countries. Socioeconomic group influences the risk of sustaining a coronary event $\mathrm{t}^{3-5}$ and is a major determinant of coronary mortality at the population level. ${ }^{6-10}$ However, information about the effects of socioeconomic deprivation on case fatality for patients with acute myocardial infarction (AMI) is limited. $^{11}$

Using education levels as a surrogate for deprivation, North American studies of AMI found a worse prognosis in the least educated people, although whether this reflected differences in health care provision could not be established. ${ }^{12}{ }^{13}$ In the UK two retrospective studies in Scotland applied Carstairs and Morris deprivation scores to infarct populations but arrived at contradictory conclusions. ${ }^{14}{ }^{15}$ One of these used the Scottish record linkage system for patients admitted to hospital with AMI and found no indication that socioeconomic deprivation affected case fatality in the first 30 days. ${ }^{14}$ The other used the Glasgow MONICA (monitoring trends and determi- nants in cardiovascular disease) coronary event register for patients under 65 and did find a socioeconomic gradient in case fatality that was more pronounced for pre-hospital than for in-hospital deaths. ${ }^{15}$

In the present study we have re-examined the effects of socioeconomic deprivation on case fatality in a prospective nine year study of patients in a hospital in east London.

\section{Methods}

The study was based on patients with AMI admitted to the coronary care unit (CCU) of Newham General Hospital between January 1988 and December 1996 (fig 1). Of the 1616 patients with AMI admitted to the hospital over this period, we excluded from analysis the 61 who were not admitted to CCU and the 39 who were not of white or south Asian background. Of the remaining 1516, we could not classify the deprivation score of 99 patients (see below), leaving a study population of 1417 patients.

The diagnosis of myocardial infarction was based on any two of the following three criteria: typical chest pain, $>0.1 \mathrm{mV} \mathrm{ST}$ elevation in at least one standard or two precordial leads, and rise in serum creatine kinase to $>400 \mathrm{IU} / 1$ (upper limit of reference range is $200 \mathrm{IU} / \mathrm{l}$ ). 


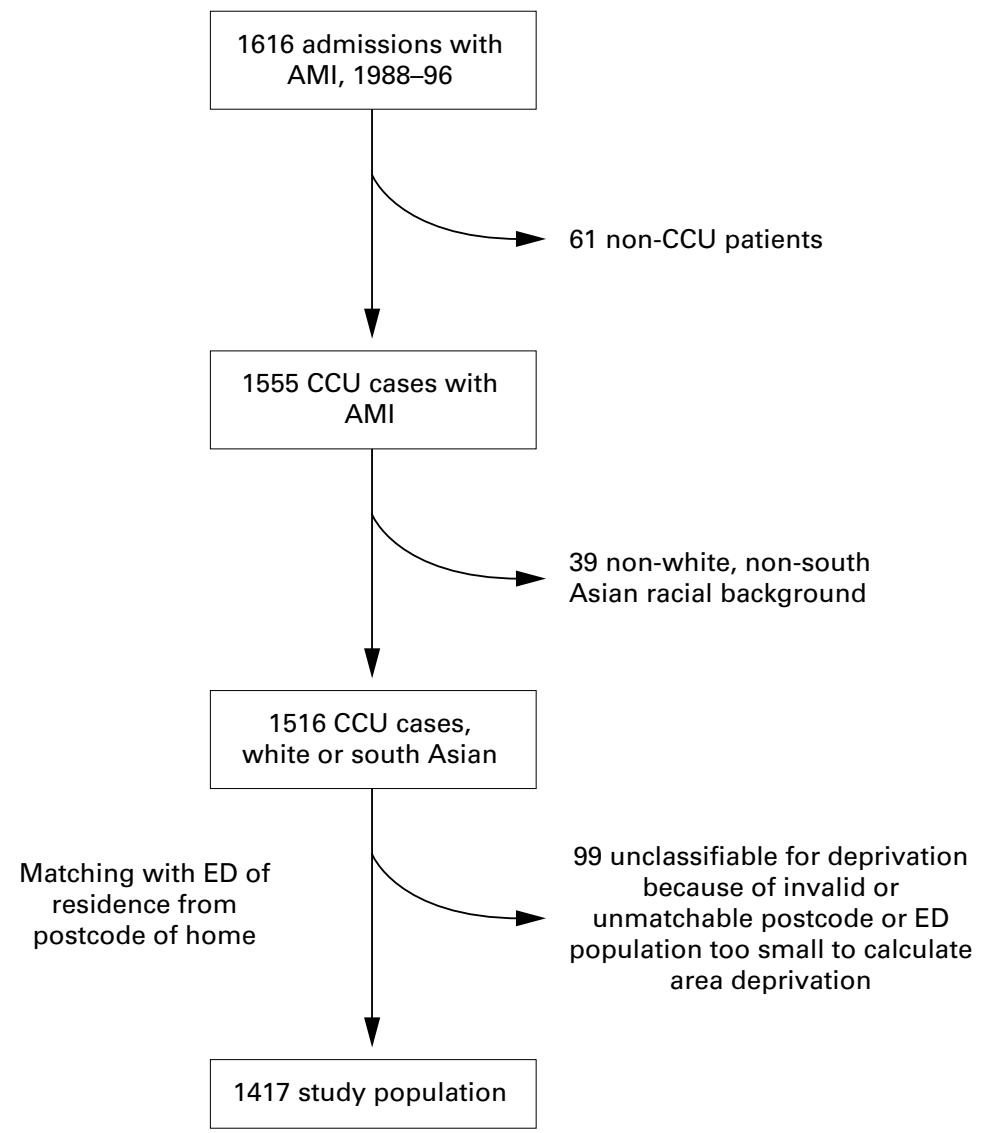

Figure 1 Selection of the study population. AMI, acute myocardial infarction; $C C U$, coronary care unit; ED, enumeration district.

Baseline clinical data were collected prospectively and stored electronically as previously described. ${ }^{16}$ Information recorded included the patient identification number and home address, details of clinical history, examination findings, electrocardiographic data, cardiac enzyme results, and details of treatment in hospital and at discharge. Information regarding race and smoking habit was obtained by direct inquiry. One of three racial groups (white, Asian, or other) was assigned by one of two clinicians (ADT or KR), where necessary by direct inquiry. A diagnosis of diabetes was recorded if the patient required insulin, oral hypoglycaemic drugs, or dietary sugar restriction. The diagnosis of left ventricular failure was recorded for patients requiring diuretic treatment in whom there were radiological signs of interstitial or alveolar pulmonary oedema, or symptoms of breathlessness accompanied by basal crepitations or a third heart sound.

The socioeconomic status of each patient was derived from Carstairs deprivation ${ }^{1}$ score of his or her enumeration district of residence. (An enumeration district on average contains around 170 households or 400 people.) The Carstairs score, a composite of four census variables-social class of head of household, overcrowding, car ownership, and unemployment-was computed from 1991 census small area data. Patients were linked to the enumeration district of residence by the full unit postcode. Deprivation scores could not be assigned to 99 of the 1516 south Asian and white patients with AMI admitted to CCU because we were unable to match the postcode or because the enumeration district population was too small to calculate an area deprivation score.

Patients were followed up for all cause mortality and recurrent ischaemic events (readmission with unstable angina, AMI, or death) from the admission date until censoring in October 1997. All the patients were flagged by the National Health Service central register to obtain follow up mortality data. For patients in whom information about non-fatal ischaemic events was not already available from outpatient follow up or readmission to hospital, postal questionnaires were used to obtain the information, backed up by telephone inquiry for non-responders. In this way, follow up data beyond discharge were obtained in $98.6 \%$ of patients who survived to hospital discharge.

STATISTICAL ANALYSIS

Tables are shown with patients grouped into quartiles of the deprivation distribution. Trends across deprivation groups were evaluated using the non-parametric test described by Cuzick ${ }^{17}$ and implemented in Stata by the nptrend command. Survival was calculated by the Kaplan-Meier method; survival probabilities are expressed as percentages with $95 \%$ confidence intervals (CI). Multivariate predictors of survival are based on a proportional hazard model. Hazard ratios (relative risks) are expressed as the increase in risk corresponding to a change from the 5 th to 95 th centile of the deprivation distribution. In broad terms, this can be interpreted as the risk of patients from the most deprived enumeration districts compared with that of patients in the least deprived enumeration districts. Significance tests for proportional hazards regression were based on comparisons of likelihood ratios.

\section{Results}

CLINICAL CHARACTERISTICS AND TREATMENT

Age and sex distributions were similar for all deprivation groups (table 1). However, the proportion of patients of south Asian origin increased from $15.1 \%$ in the least deprived to $46.1 \%$ in the most deprived quartile, and the proportion of patients with diabetes from $16.6 \%$ to $28.9 \%$. There was no evidence of a significant trend with deprivation in smoking habit, hypertension, or other risk factors.

The time from pain onset to hospital arrival was similar for all deprivation groups, as was emergency treatment received in hospital, there being no differences among the groups in proportions receiving aspirin and thrombolysis, or in the time taken to administer thrombolysis.

There were no differences among deprivation groups in infarct location. However, Q wave infarction increased with socioeconomic deprivation, as did left ventricular failure, but not ventricular fibrillation. A suggestive but not significant trend with deprivation was seen in hospital mortality.

Proportions of patients discharged on aspirin and beta-blockers were similar for all groups. 
Table 1 Patient characteristics, treatment, and clinical complications by quartile (Q1-Q4) of the Carstairs deprivation score

\begin{tabular}{|c|c|c|c|c|c|c|c|c|}
\hline & \multicolumn{7}{|c|}{ Median (interquartile range) or count (\%) } & \multirow[b]{2}{*}{$\begin{array}{l}p \text { Value } \\
\text { for treno }\end{array}$} \\
\hline & \multicolumn{2}{|c|}{$\begin{array}{l}\text { Q1 (least deprived) } \\
(n=358)\end{array}$} & \multicolumn{2}{|c|}{$\underset{(n=351)}{Q 2}$} & $\underset{(n=354)}{Q 3}$ & \multicolumn{2}{|c|}{$\begin{array}{l}Q 4 \text { (most deprived) } \\
(n=354)\end{array}$} & \\
\hline \multicolumn{9}{|c|}{ Characteristics at presentation } \\
\hline Age (years) & 64 & $(55-72)$ & 64 & $(56-73)$ & $62(54-71)$ & 62 & $(55-71)$ & $>0.2$ \\
\hline Male & 269 & $(75.1 \%)$ & 243 & $(69.2 \%)$ & $261(73.7 \%)$ & 272 & $(76.8 \%)$ & $>0.2$ \\
\hline Diabetes & 59 & $(16.6 \%)$ & 66 & $(18.9 \%)$ & $72(20.4 \%)$ & 102 & $(28.9 \%)$ & $<0.01$ \\
\hline White & 304 & $(84.9 \%)$ & 304 & $(86.6 \%)$ & $260(73.5 \%)$ & 191 & $(54.0 \%)$ & \\
\hline Asian & 54 & $(15.1 \%)$ & 47 & $(13.4 \%)$ & $94(26.6 \%)$ & 163 & $(46.1 \%)$ & $<0.01$ \\
\hline Non-smoker & 67 & $(19.1 \%)$ & 59 & $(17.3 \%)$ & $68(19.7 \%)$ & 69 & $(19.9 \%)$ & \\
\hline Ex-smoker & 117 & $(33.4 \%)$ & 99 & $(29.0 \%)$ & $85(24.6 \%)$ & 123 & $(35.6 \%)$ & 0.14 \\
\hline Smoker & 166 & $(47.4 \%)$ & 183 & $(53.7 \%)$ & $192(55.7 \%)$ & 154 & $(44.5 \%)$ & \\
\hline Hypertension & 128 & $(36.2 \%)$ & 100 & $(28.8 \%)$ & $86(24.6)$ & 121 & $(34.4)$ & $>0.2$ \\
\hline Previous MI & 81 & $(22.8 \%)$ & 74 & $(21.2 \%)$ & $87(24.6 \%)$ & 84 & $(23.8 \%)$ & $>0.2$ \\
\hline Pain duration & 115.5 & $(65-240)$ & 120 & $(67-240)$ & $120(60-251)$ & 120 & $(68-243)$ & $>0.2$ \\
\hline Anterolateral & 188 & $(53.3 \%)$ & 168 & $(48.5 \%)$ & $172(49.0 \%)$ & 182 & $(51.9 \%)$ & \\
\hline Inferoposterior & 165 & $(46.7 \%)$ & 178 & $(51.5 \%)$ & $179(51.0 \%)$ & 169 & $(48.2 \%)$ & $>0.2$ \\
\hline $\mathrm{Q}$ wave & 263 & $(75.1 \%)$ & 241 & $(69.3 \%)$ & $292(83.7 \%)$ & 284 & $(80.9 \%)$ & $<0.01$ \\
\hline \multicolumn{9}{|l|}{ Treatment } \\
\hline Time to lysis & 80 & $(54-160)$ & 81. & $(52-149)$ & $77(57-123)$ & 89. & $(55-140)$ & $>0.2$ \\
\hline Aspirin (acutely) & 313 & $(89.4 \%)$ & 316 & $(91.6 \%)$ & $312(89.1 \%)$ & 317 & $(90.3 \%)$ & $>0.2$ \\
\hline Thrombolysis & 262 & $(73.1 \%)$ & 259 & $(73.8 \%)$ & $262(74.0 \%)$ & 268 & $(75.7 \%)$ & $>0.2$ \\
\hline \multicolumn{9}{|l|}{ Complications } \\
\hline LVF & 105 & $(29.5 \%)$ & 108 & $(31.0 \%)$ & $127(36.0 \%)$ & 124 & $(35.2 \%)$ & 0.05 \\
\hline VF & 41 & $(11.6 \%)$ & 23 & $(6.3 \%)$ & $41(11.7 \%)$ & 23 & $(6.5 \%)$ & 0.13 \\
\hline Hospital death & 42 & $(11.7 \%)$ & 44 & $(12.6 \%)$ & $48(13.6 \%)$ & 56 & $(15.8 \%)$ & 0.1 \\
\hline \multicolumn{9}{|l|}{ Discharge medication } \\
\hline Aspirin & 277 & $(91.1 \%)$ & 271 & $(91.3 \%)$ & $267(91.4 \%)$ & 272 & $(94.1 \%)$ & 0.2 \\
\hline$\beta$ Blockers & 136 & $(44.7 \%)$ & 119 & $(40.6 \%)$ & $103(35.2 \%)$ & 122 & $(42.1 \%)$ & $>0.2$ \\
\hline
\end{tabular}

Data were missing or not applicable in the following numbers of patients: age (3), diabetes (5), smoking status (35), hypertension (4), previous acute myocardial infarction (MI) (5), pain duration (99), site of infarction (4), Q wave infarction (19), time to thrombolysis (463), (acute) aspirin treatment (21), left ventricular failure (LVF) (7), ventricular fibrillation (VF) (10), hospital death (1), discharge aspirin (235), discharge $\beta$ blockers (237).

Table 2 Kaplan-Meier event free survival probabilities (95\% confidence intervals) by quartile (Q1-Q4) of the Carstairs deprivation score

\begin{tabular}{lllll}
\hline & $\begin{array}{l}Q 1 \text { (least deprived) } \\
(n=358)\end{array}$ & $Q 2(n=351)$ & $Q 3$ (n=354) & $\begin{array}{l}\text { Q4 (most deprived) } \\
(n=354)\end{array}$ \\
\hline $\begin{array}{l}\text { Recurrent ischaemic events (including death) } \\
\quad \text { 3 days }\end{array}$ & $0.85(0.80$ to 0.88$)$ & $0.83(0.78$ to 0.86$)$ & $0.83(0.78$ to 0.86$)$ & $0.79(0.74$ to 0.83$)$ \\
6 months & $0.75(0.70$ to 0.79$)$ & $0.74(0.69$ to 0.78$)$ & $0.73(0.68$ to 0.76$)$ & $0.70(0.65$ to 0.75$)$ \\
1 year & $0.70(0.65$ to 0.75$)$ & $0.65(0.60$ to 0.70$)$ & $0.70(0.65$ to 0.75$)$ & $0.64(0.59$ to 0.69$)$ \\
3 years & $0.58(0.52$ to 0.63$)$ & $0.50(0.44$ to 0.55$)$ & $0.56(0.50$ to 0.61$)$ & $0.53(0.47$ to 0.58$)$ \\
Death & & & & \\
30 days & $0.88(0.84$ to 0.91$)$ & $0.87(0.82$ to 0.90$)$ & $0.86(0.82$ to 0.89$)$ & $0.82(0.77$ to 0.85$)$ \\
6 months & $0.84(0.79$ to 0.87$)$ & $0.81(0.76$ to 0.85$)$ & $0.82(0.77$ to 0.85$)$ & $0.79(0.74$ to 0.83$)$ \\
1 year & $0.81(0.77$ to 0.85$)$ & $0.76(0.71$ to 0.80$)$ & $0.80(0.76$ to 0.84$)$ & $0.77(0.72$ to 0.81$)$ \\
3 years & $0.72(0.66$ to 0.76$)$ & $0.65(0.59$ to 0.70$)$ & $0.69(0.63$ to 0.74$)$ & $0.72(0.66$ to 0.76$)$ \\
\hline
\end{tabular}

SURVIVAL ANALYSIS

At 30 days, event free survival for both recurrent ischaemic events and mortality fell progressively with deprivation: for recurrent ischaemia from 0.85 (95\% CI 0.80 to 88 ) in the least deprived quartile to 0.79 (95\% CI 0.74 to 0.83 ) in the most deprived quartile, and for mortality from 0.88 (95\% CI 0.84 to 0.91 ) to 0.82 (95\% CI 0.77 to 0.85 ) (table 2). But the deprivation gradient tended to flatten over time (fig 2). Because of the time dependent change in relative risk, we confined further analyses of survival to the first year of follow up, and analysed survival separately over the first 30 days and from day 30 to one year.

MULTIVARIATE ANALYSIS

The hazard ratios (corresponding to an increase from the 5th to 95th centile of the deprivation distribution) suggested that patients from more deprived enumeration districts were at significantly greater risk of recurrent ischaemic events over the first 30 days (unadjusted hazard ratio 1.54 (95\% CI 1.02 to 2.32 ) (table 3). Point estimates and confidence intervals were little altered by adjustment for age, sex, racial group, diabetes, acute thrombolytic and aspirin treatment, and left ventricular failure. Significance was lost on additional adjustment for discharge drugs (aspirin and $\beta$ blockers), though this result was based on the smaller number of patients who survived to discharge and had complete data on discharge medication; in fact the point estimate increased slightly. From 30 days to one year, however, there was no evidence that the frequency of recurrent ischaemic events increased with deprivation, suggesting that the period of increased risk associated with deprivation was short lived. A similar pattern was seen with mortality.

\section{Discussion}

Our prospective study provides new insights into the relation between socioeconomic deprivation and the short and medium term outcomes of patients admitted to hospital with AMI. The data, which include all age groups, show a clear socioeconomic gradient in recurrent ischaemia and mortality in the first month of AMI, which then appears to be attenuated over time. 
because survival differences among the deprivation groups had largely disappeared by one year. This assumption is supported by the Glasgow MONICA data, which showed that the socioeconomic gradient was more pronounced for pre-hospital deaths than for deaths among patients admitted to hospital. ${ }^{15}$ It is well established that, in AMI, deaths in the pre-hospital phase are usually arrhythmic while deaths in the hospital phase are usually haemodynamic, caused by extensive myocardial injury. ${ }^{21}{ }^{22}$ There is unlikely, therefore, to be any simple explanation for the effect of deprivation because it appears to affect both phases of early outcome. Nevertheless, it may be significant that the point estimates of risk for the most deprived group had fallen below those of the least deprived group a year after myocardial infarction, suggesting that some of the excess early risk might reflect a "harvesting" phenomenon related to the impact of myocardial infarction, in addition to the debilitation that commonly accompanies severe deprivation such as nutritional deficiency, ${ }^{23}$ concomitant illness ${ }^{24}$ or autonomic disturbance. ${ }^{25}$

In conclusion, our findings provide further evidence of the vital interaction between socioeconomic status and health. They make clear that in patients hospitalised with AMI, early case fatality increases with increasing levels of deprivation. Add to this the well established relation between socioeconomic group and the population mortality from coronary heart disease and it is evident that inequalities in health continue to run deep in society. No simple or treatable mechanism is likely to emerge that accounts for the adverse effect of deprivation on coronary mortality, and its resolution in the long term will almost certainly depend on implementation of effective strategies to reduce poverty.

1 Carstairs V, Morris R. Deprivation and health in Scotland. Aberdeen: Aberdeen University Press, 1991.

2 Davey Smith G, Shipley MJ, Rose G. The magnitude and causes of socioeconomic differentials in mortality: further evidence from the Whitehall study. $\mathcal{F}$ Epidemiol Community Health 1990;44:265-70.
3 Hebert PR, Buring JE, Rosner B, et al. Occupation and risk of of nonfatal myocar

4 Hammar N, Alfredsson L, Smedberg M, et al. Differences in the incidence of myocardial infarction among occupational groups. Scand f Work Environ Health 1992;18:178-85.

5 Smith WCS, Kenicer MB, Tunstall-Pedoe $\mathrm{H}$, et al. Prevalence of coronary heart disease in Scotland; Scottish heart health study. Br Heart f 1990;64:295-8.

6 Holme I, Helgeland A, Hjermann I, et al. Four-year mortality by some socio-economic indicators: the Oslo study. F Epidemiol Community Health 1980;34:48-52.

7 Pearce NE, Davis PB, Smith AH, et al. Mortality and social class in New Zealand II: male mortality by major disease groupings. NZ Med f 1983;96:711-6.

8 Marmot MG, Shipley MJ, Rose G. Inequalities in death: specific explanations of a general pattern. Lancet 1984; $1003-6$.

9 Rosengren A, Wedel H, Wilhelmsen L. Coronary heart disease and mortality among men from different occupational classes in Sweden. Br Heart f 1988;297:1497-500.

10 Marmot MG, McDowall MG. Mortality decline and widening social inequalities. Lancet 1986;ii:274-6.

11 Wilhelmsen L, Rosengren A. Are there socio-economic differences in survival after acute myocardial infarction? Eur Heart F 1996;17:1619-23.

12 Weinblatt E, Ruberman W, Goldberg JD, et al. Relation of education to sudden death after myocardial infarction. $N$ Engl f Med 1978;299:957-62.

13 Tofler GH, Muller JE, Stone PH, et al. Comparison of longterm outcome after acute myocardial infarction in patients never graduated from high school with that in more educated patients. Am f Cardiol 1993;71:1031-5.

14 Capewell S, Kendrick S, Boyd J, et al. Measuring outcomes: one month survival after acute myocardial infarction in Scotland. Heart 1996;76:70-5.

15 Morrison C, Woodward m, Leslie W, et al. Effect of socioeconomic group on incidence of, management of, and survival after myocardial infarction and coronary death: analysis of community coronary event register. BMF 1997; analysis of

16 Stevenson R, Ranjadayalan K, Wilkinson P, et al. Short and long term prognosis of acute myocardial infarction since introduction of thrombolytic therapy. BMF 1993;307:34953

17 Cuzick J. A Wilcoxon-type test for trend. Stat Med $1985 ; 4: 87-90$

18 Wilkinson P, Sayer J, Laji K, et al. Comparison of case fatality in south Asian and white patients after acute myocardial infarction. BMF 1994;312:1330-3.

19 Gibson RS. Non-Q-wave myocadial infarction. In: Fuster V, Ross R, Topol EJ, eds. Atherosclerosis and coronary artery disease. Philadelphia: Lippincott Raven, 1996:1097.

20 Anon. Health in the East End: annual public health report 1998/99. London: East London and The City Health Authority, 1999 .

21 Antman EM, Rutherford JD Coronary care medicine. Boston: Martinus Nijhoff, 1986:20.

22 Braunwald E. Heart disease: a textbook of cardiovascular medicine. Philadelphia: WB Saunders.

23 Schettler G. Atherosclerosis during periods of food deprivation following world wars I and II. Prev Med 1983;12:7583.

24 Hawthorne VM, Watt GCM, Hart CL, et al. Cardiorespiraory and all cause mortality in men and women in urban Scotland; 15 year follow up. Scott Med f 1995;40:102-7.

25 Ruberman W. Psychosocial influences on mortality of patients with coronary heart disease. $7 A M A$ 1992;267: 559-60. 